\title{
Fracture of particulate composites
}

\begin{abstract}
S RAY
Department of Metallurgical Engineering, University of Roorkee, Roorkee 247672, India

Abstract. A thermodynamic analysis of the process of fracture in elastically deformable composites is formulated. The critical dimensions leading either to particle fracture or to matrix-particle decohesion are identified. Fracture in plastically deformable composites is discussed in the light of the experimental evidence regarding void or cavity nucleation. Models of void growth under the application of stress and the role of void growth in causing failure are described in brief.
\end{abstract}

Keywords. Fracture; composite; void nucleation; void growth; cavity.

\section{Introduction}

Particulate composites are materials containing one or more discontinuous phases dispersed in a matrix of a distinctly different phase, such that the length to diameter ratio of each dispersoid is of the order of unity. If the extent of the discontinuous phase (or phases) is small and the load shared by it is insignificant, the material is not effectively regarded as a composite because the mechanical property of the material does not reflect the characteristics of the discontinuous phase. However, many materials that are not originally designed as particulate composites may pick up discontinuous phases during processing in the form of particles of insoluble impurities or inclusions which subsequently exert an important influence on the fracture behaviour of these materials. Particulate composites can be divided into four basic classes depending on the mechanical properties of the continuous phase (the matrix) and the discontinuous phase (the particle). (a) brittle matrix-brittle particle, (b) brittle matrix-ductile particle, (c) ductile matrix-brittle particie, and (d) ductile matrix-ductile particle. The fracture behaviour of the first two classes will be analysed under the broad heading of elastically deformable composites in $\S 2$, and fracture in the other two classes will be examined in $\S 3$.

The first systematic approach to the process of fracture was developed by Griffith (1920) to explain the discrepancy between the ideal strength and the observed strength in a brittle material. The thermodynamics of the process of extension of an atomically sharp pre-existing crack of length $2 c$ under an applied stress of $\sigma$ was examined. When a crack extends, elastic energy is released due to relaxation of stress around the extended part of the crack; some energy is also expended by the system in creating a fresh crack surface. The extension of the crack becomes feasible only when the former energy exceeds the latter, thereby reducing the total free energy. The fracture stress, $\sigma_{0}$, calculated on the above basis is as given below.

$$
\sigma_{0}=(2 \alpha E / \pi c)^{1 / 2}
$$

where $\alpha$ is the specific energy of the crack surface and $E$ is the Youngs modulus. 
Orowan (1949) provided a similar analysis for the extension of a crack under the plane stress condition. Sack (1946) and Sneddon (1946) modified the estimate of $\sigma_{0}$ in (1) by including the stress concentration effect around the crack. However, the original approach is based on the very general principles of thermodynamics and so provides a necessary criterion for the propagation of a crack leading to fracture. Here, we use this simple approach to examine the different possible fracture processes in the problem at hand, to gain an insight into the underlying essentials. Detailed stress calculations will not be of help in this regard because crack propagation is controlled by the local stress and strain which are inhomogeneous and dependent on the exact particle shape and distribution. These material parameters vary considerably from sample to sample, even for the same engineering material.

\section{Elastically deformable composites}

Any composite with a brittle matrix can be deformed only elastically irrespective of the nature of the reinforcing particle. To determine the fracture behaviour it is necessary to analyse the local stress distribution around the particles. When a particulate composite is deformed, the force is not directly applied to the particles but is transferred through the matrix. We consider the simple model of a particle embedded in a brittle matrix as given in figure 1 . To simplify the mathematical treatment the following assumptions are made: (i) Poisson's effect is negligible; (ii) both the particle and the matrix have the same shear modulus. The elastic matrix is subjected to a tensile stress of $\sigma_{m}$. The forces acting on an element extending an angle $d \theta$ at the centre are shown in figure $1(\mathrm{~b})$. If $\tau_{\theta}$ is the shear stress acting at the particle-matrix interface, the elemental force balance in the direction of the applied stress gives

$$
\mathrm{d} \sigma / \mathrm{d} \theta=2 \tau_{\theta} .
$$

A special case (i.e., equal elastic moduli for particle and matrix) of the general result derived by Sezawa and Miyazaki (1928) then yields, for the magnitude of the stress distribution $\tau_{\theta}$ at the particle-matrix interface, the expression

$$
\tau_{\theta}=(1 / 2) \sigma_{m} \sin 2 \theta \text {. }
$$

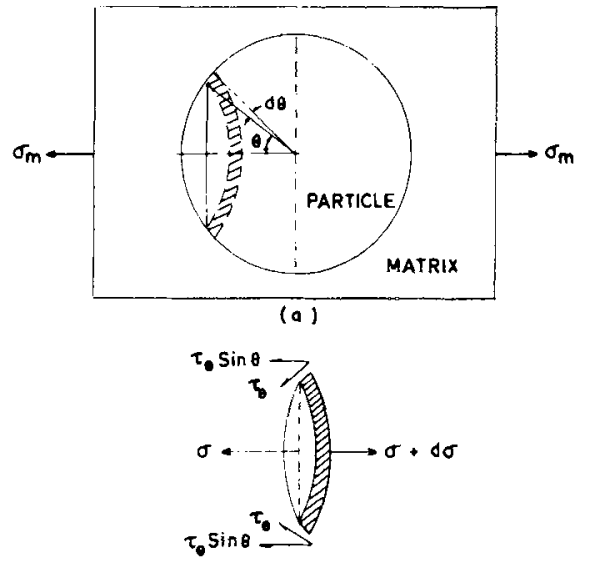

(b)
Figure 1. (a) Schematic representation of a single particle composite model. (b) Elemental force balance at a section of the particle. 
Inserting this in (2) and integrating, the magnitude of the stress in the particle at a plane labelled by the angie $\theta$ is

$$
\sigma_{p}=\sigma_{m} \sin ^{2} \theta
$$

Let $\bar{\sigma}_{m}, \bar{\sigma}_{p}$ and $\bar{\tau}$ be the fracture strengths of the matrix, particle and interface. Then the matrix, or the particle, or the interface fractures first, if $\bar{\sigma}_{m}$, or $\bar{\sigma}_{p}$, or $2 \bar{\tau}$ is the smallest of the three quantities $\bar{\sigma}_{m}, \bar{\sigma}_{p}, 2 \bar{\tau}$. In the first case, the original analysis of Griffith and its subsequent modifications are applicable, after the inhomogeneous stress distribution is determined. In the second and third cases, a similar analysis brings out certain additional parameters of interest.

\subsection{Particle fracture}

When the particle has fractured at a plane whose angle of extension is $2 \theta$ at the centre, this crack will be able to propagate in the matrix provided it is thermodynamically feasible. If the particle has a radius $r$, the change in energy $\Delta E$ for an infinitesimal extension $\mathrm{d} r$ of the crack is given by

$$
\Delta E=-4 \pi r^{2} \mathrm{~d} r \sigma_{m}^{2}\left(1-v_{m}\right)^{2} \sin ^{2} \theta / E_{m}+2 \pi r \mathrm{~d} r \gamma_{m} \sin \theta
$$

where $v_{m}$ and $E_{m}$ are Poisson's ratio and the elastic constant respectively of the matrix and $\gamma_{m}$ is the surface energy of the matrix. For the crack to propagate spontaneously, $\Delta E$ should be negative, which implies that

$$
r>r_{c}=E_{m} \gamma_{m} /\left[2\left(1-v_{m}\right)^{2} \sigma_{m}^{2} \sin ^{2} \theta\right] .
$$

Since the particle has fractured when $\sigma_{p}=\bar{\sigma}_{p}=\sigma_{m} \sin ^{2} \theta$ the composite will at once satisfy the necessary condition for failure if

$$
r>E_{m} \gamma_{m} \sin ^{3} \theta /\left[2\left(1-v_{m}\right)^{2} \tilde{\sigma}_{p}^{2}\right] .
$$

Otherwise, the load can be increased till it attains the value

$$
\sigma_{m}=\left[E_{m} \gamma_{m} / 2 r\left(1-v_{m}\right)^{2} \sin ^{2} \theta\right]^{1 / 2} \text {. }
$$

The matrix will fail at a load different from that causing the fracture of the particles. As a range of particle size is involved in real-life composites, the estimate of $\sigma_{m}$ given by (8) must be calculated using the largest value of the particle radius $r$ that is involved.

\subsection{Interface fracture}

When the interface is weak, let a crack be initiated there at an angle $\theta$. The crack will extend along the interface from an angle $\theta$ to $\theta+\mathrm{d} \theta$ if the corresponding change in energy is negative. This change is given by

$$
\Delta E=-\pi r^{3} \sigma_{m}\left(1-v_{p}\right)^{2} \sin ^{4} \theta \mathrm{d} \theta / E_{p}+2 \pi r^{2}\left(\gamma_{p}+\gamma_{m}-\gamma_{p m}\right) \sin \theta \mathrm{d} \theta
$$

where $v_{p}$ and $E_{p}$ are Poisson's ratio and the elastic constant, respectively, of the particle; $\gamma_{p}$ is the surface energy of the particle and $\gamma_{p m}$ is the particle-matrix interfacial energy. The debonding between the matrix and the particle will take place as long as $\Delta E<0$, and will extend up to

$$
\theta_{c}=\sin ^{-1}\left[\frac{2\left(\gamma_{p}+\gamma_{m}-\gamma_{p m}\right) E_{p}}{r\left(1-v_{p}\right)^{2} \sigma_{m}}\right]^{1 / 2}
$$


Clearly, this crack can propagate into the matrix if the size of the particle is such that

$$
r \geqslant E_{m} \gamma_{m} /\left[2\left(1-v_{m}\right)^{2} \sigma_{m}^{2} \sin \theta_{c}\right] .
$$

If $r$ is smaller than the quantity on the right, the load must be increased to the level given by $(8)$ in order to fracture the matrix.

The surface energy of the particle, that of the matrix, and the particle-matrix interfacial energy play a vital role in determining the mode of fracture. The experimental determination of these energies is difficult and sometimes unreliable because of the segregation of impurities. The predictions of the above analysis may also become unreliable due to kinetic factors.

\section{Plastically deformable composites}

The phenemenon of fracture in a composite with a ductile matrix is commonly encountered in metals and alloys with inclusions. As composites contain a combination of phases, it is instructive to explore first the mechanism of fracture in a ductile singlephase material.

It is observed that cavities form in front of propagating cracks. It has therefore been assumed that vacancies in the highly strained region condense into voids. Under appropriate conditions of stress, these voids give rise to propagating cracks leading to the failure of the material. However, Balluffi and Siegel (1955) contend that the excess concentration of vacancies produced due to strain is not sufficient to lead to the homogeneous nucleation of voids, and that nuclei for void formation must pre-exist in the specimen. It is well known that cavities may also nucleate through a host of other mechanisms. For example, grain boundary sliding at low strain rates and intermediate temperatures gives rise to voids; and under such circumstances any factors preventing grain boundary sliding would also suppress the nucleation of cavities. Smith and Barnby (1967) have observed the nucleation of cavities at dislocation pile-ups, while Davies and Williams (1969) have observed this process taking place at twin-grain boundary intersections. All these mechanisms are operative at sites with large stress concentrations.

Such stress concentrations exist in the neighbourhood of the particles in a composite, and cavities form there either by cavity nucleation as in pure materials, or by mechanical decohesion of the matrix-particle interface as explained in $\S 2$. Thermodynamic analysis (Greenwood and Harris 1965) shows that if $\gamma_{p m}<10^{-3} \gamma_{m}$, mechanical decohesion is the most likely mode for cavity formation. Brown and Stobbs (1971) have estimated the critical strain for mechanical decohesion. A comparison of this estimate with the experimental value observed in a composite of silica particles dispersed in copper (Palmer and Smith 1968) indicates that the theoretical value is highly overestimated. This poses a problem, because a thermodynamic analysis provides necessary conditions and therefore minimal estimates in general. The discrepancy here may be attributed to the relaxation of stress observed around the smaller particles by the formation of prismatic loops. However, no such stress relaxation occurs around large particles and voids are readily formed there. Given a detailed understanding of the mechanisms of void creation, no quantitative theory of the fracture process under consideration is available, because the process is ultimately controlled by local stresses and strains, which in turn are governed by the shape and distribution of the particles in 
the matrix. Moreover, once voids are formed, the stress induces further growth and both the volume and the shape of the voids change, with the actual strain history exerting a significant influence.

Mcclintock (1968) proposed the first model for the growth of a cylindrical hole in a hardening plastic matrix. Subsequently, Rice and Tracey (1969) investigated another model for the growth of a spherical hole in a non-hardening plastic matrix and proposed a nonlinear relation between the radial velocity of the void surface and the applied strain field. Jalinier (1981) applied the linear version of the model to the damage observed in sheet metal. For a void caused by the decohesion of the particle-matrix interface as well as for a void generated by the cracking of the particle, $\varepsilon_{i}=B_{i} \varepsilon_{i}^{\infty}$, where $\varepsilon_{i}$ is the relative extension of the void in the $i$-direction and $\varepsilon_{i}^{\infty}$ is the applied strain field far from the void. For decohesion, $B_{i}$ is a function of the ratio $\varepsilon_{2} / \varepsilon_{1}$ of the principal strains, which for fragmentation is a constant $\approx 4$. These theoretical results agree well with experimental observations.

The growth of the voids leads to failure by two possible mechanisms. The material between growing voids starts getting thinner till a necking instability sets in, resulting in the coalescence of the voids and eventual fracture. It is also possible that at some stage in the growth of the voids, conditions become ripe for cracks to originate from the voids, as explained in $\S 2$.

\section{Conclusions}

Fracture in particulate composites is an important area in need of viable theories. The difficulties arise from the complexity of the stress distribution in an inhomogeneous material and the dependence of the fracture process on highly local stress and strain conditions. Estimates based on energy considerations have led to some understanding of the phenomena involved, but much remains to be done.

\section{References}

Balluffi R W and Siegel L L 1955 Acta Metall. 3170 Brown L M and Stobbs W M 1971 Philos. Mag. 231201

Davies P W and Williams K R 1969 J. Metal Sci. 348

Greenwood G W and Harris J E 1965 Acta Metall. 13936

Griffith A A 1920 Philos. Trans. R. Soc. A221 163

Jalinier J M 1981 Thesis, Univ. of Metz, France (unpublished)

Mcclintock F A 1968 J. Appl. Mech. 35363

Orowan E 1949 Rep. Prog. Phys. 12185

Palmer I G and Smith G C 1968 Oxide dispersion strengthening (New York: Gordon and Breach) p 253

Rice J R and Tracey D M 1969 J. Med. Phys. Solids 17201

Sack R A 1946 Proc. Phys. Soc. 58729

Sezawa K and Miyazaki B 1928 Proc. Jpn Soc. Mech. Eng. 31625

Smith E and Barnby J T 1967 J. Metal Sci. 11

Sneddon I N 1946 Proc. R. Soc. A187 229

\section{Discussion}

P Rodriguez: Why has the dislocation pile-up at the interface not been taken into account? 
S Ray: Dislocation pile-up treatments are suspect. All these effects have been put into an experimental parameter $\varepsilon_{m}$.

V C Sahni: What is the implication if you have an interface of finite thickness?

Ray: I do not know the answer at present.

$G$ Srinivasan: Is there an essential difference between the way a crack propagates in metals and insulators on the one hand, and in crystalline and amorphous materials on the other?

Ray: There is no appreciable difference.

K Srinivasa Raghavan: What about the possibility that the crack nucleates at the interface and goes into the matrix or the particle as decided by the relative moduli? Ray: It is possible to treat this case along the same lines, but my interest has been in the problem of incipient cracking of the particle. 